\title{
Antidementivum bewährt sich auch im Praxisalltag
}

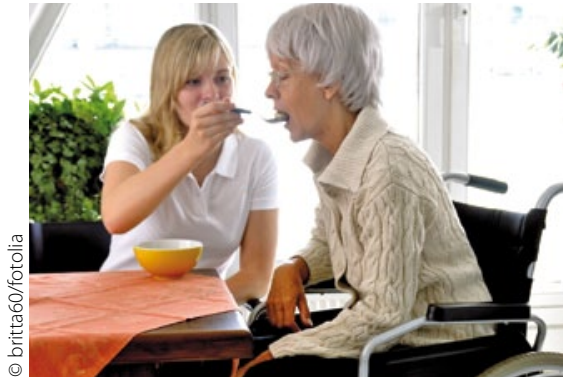

Bei einem Drittel der Patienten verringerte sich der Betreuungsaufwand.

- Für das Antidementivum Memantine liegen nicht nur gute Daten aus placebokontrollierten Studien vor - das Medikament kann auch unter Praxisbedingungen Alzheimerpatienten stabilisieren und den Betreuungsaufwand senken.

Für Memantine (z. B. Axura ${ }^{\oplus}$ ) ist in zahlreichen kontrollierten Studien ein Nutzen bei moderater bis schwerer Alzheimer-Demenz belegt worden. In einer Metaanalyse von sechs Studien mit 2300 Patienten waren die kognitiven Fähigkeiten bei modera- ter Demenz nach 24 Wochen Therapie sogar besser als zu Beginn, der ADAS-cogWert war dabei im Schnitt etwa 1,5 Punkte besser als mit Placebo, und bei schwerer Demenz lag der Wert auf der Severe Impairment Battery 3-4 Punkte höher als mit Placebo (Int J Geriatr Psychiatry 2009; 24: 532). Daran erinnerte Prof. Matthias Riepe, UIm. Allerdings werden in Studien oft Teilnehmer mit hohem Alter oder Komorbiditäten ausgeschlossen, sodass sie nicht unbedingt mit denen in der Praxis vergleichbar sind.

In einer aktuellen multizentrischen Untersuchung wurde die Wirksamkeit und Verträglichkeit von Memantine unter Alltagsbedingungen geprüft, bei 1325 Heimbewohnern mit einer moderaten Alzheimer-Demenz. $85 \%$ von ihnen hatten mindestens eine Begleiterkrankung, am häufigsten waren psychiatrische Störungen gefolgt von Herz-Kreislauf-Erkrankungen. Das Durchschnittsalter lag bei 79 Jahren. Alle Patienten erhielten 24 Wochen lang Memantine, im Schnitt 18 mg/d.
Nach sechs Monaten hatte sich die kognitive Funktion - gemessen mit dem MiniMental-Status-Test (MMST) - bei 35\% der Patienten gebessert, bei $60 \%$ blieb sie stabil und nur bei $5 \%$ wurde sie schlechter. Ähnliche Werte zeigten sich bei der Betreuungszeit: Sie verringerte sich bei einem Drittel, bei $60 \%$ blieb sie konstant und bei $6 \%$ nahm sie zu. In ihrem Globalurteil attestierten die befragten Ärzte 79\% der Patienten einen besseren Zustand als zu Studienbeginn, bei $14 \%$ beurteilten sie inn als stabil, nur bei 7\% nahmen sie eine Verschlechterung wahr. Die Verträglichkeit der Therapie wurde bei $98 \%$ der Patienten als sehr gut oder gut bewertet. Nach Auffassung von Riepe zeigen die Daten, dass es sich auch lohnt, ältere Patienten in Pflegeheimen antidementiv zu behandeln.

- Thomas Müller

Quellen: Satellitensymposium „AlzheimerDemenz - Status quo, Herausforderungen und Ausblicke" auf dem DGPPN-Kongress, Berlin, November 2011 (Veranstalter: Merz)

\section{Rheumatoide Arthritis}

\section{Morgendliche Funktionseinschränkungen gezielt behandeln}

— Mehr als die Hälfte der Patienten, die unter rheumatoider Arthritis leiden, fühlen sich durch morgendliche Funktionseinschränkungen in ihrer Aktivität behindert, berichtete Dr. Lothar Meier, Hofheim, der die Ergebnisse einer internationalen Erhebung vorstellte. Dabei wurde mangelnde Beweglichkeit noch störender empfunden als Schmerz. Während die befragten Ärzte den Anteil der betroffenen Patienten richtig bewerteten, unterschätzten sie die Dauer der Symptomatik. Diese wurde von rund der Hälfte der Betroffenen mit 1-2 Stunden angegeben, während viele Ärzte von 30-60 Minuten ausgingen.

\section{Chronobiologie beeinflusst Therapieerfolg}

Doch nicht nur die Bedeutung der morgendlichen Funktionseinschränkung wird bisweilen falsch beurteilt, auch die medikamentöse Therapie ist laut Meier unzureichend. Während am häufigsten einfache gymnastische Übungen empfohlen werden, gaben nur 54\% der befragten Rheumatologen an, die Morgensteifigkeit gezielt medikamentös zu behandeln. Sofern dies der Fall war, wurden fast durchgehend Glukokortikoide eingesetzt. Entscheidend ist bei dieser sinnvollen Option, dass das Medikament seine Wirkung zum richtigen Zeitpunkt entfaltet. Gut handhabbar ist in dieser Hinsicht Lodotra ${ }^{\circledR}$, das seinen Wirkstoff Prednison vier Stunden nach Einnahme freisetzt. Wird das Präparat z.B. um 22 Uhr eingenommen, entfaltet sich die Wirkung zum chronobiologisch optimalen Zeitpunkt.

Dass dies einen messbaren Einfluss auf die "Patient Reported Outcomes" hat, wie
Dr. Rieke Alten, Berlin, betonte, konnte in doppelblinden, randomisierten Studien wie z.B. CAPRA-1 nachgewiesen werden. Nach zwölf Wochen verkürzte Prednison mit modifizierter Freisetzung (MR Prednison) die Dauer der Morgensteifigkeit um 44 Minuten gegenüber konventionellem Prednison (Buttgereit F et al. Lancet 2008; 371: 205-14). Nach Abschluss der nachfolgenden offenen Phase war die Morgensteifigkeit um ca. $50 \%$ reduziert (Ann Rheum Dis 2010; 69 (7): 1275-80). Hinsichtlich unerwünschter Effekte unterschieden sich die beiden Behandlungsarme nicht.

\footnotetext{
- Dr. Christoph Posch

Quellen: Fachpressegespräch „Mehr Bewegung, mehr Lebensqualität: Neue Perspektiven für die RA-Therapie", DGRh-Kongress, München, September 2011 (Veranstalter: Mundipharma)
} 